\title{
Effects of Addition of Prosopis Juliflora Fiber on the Physical and Mechanical Properties of Wood Dust and Coir Pith Particle Reinforced Phenol Formaldehyde Hybrid Composite
}

\author{
A.Sujin Jose ${ }^{1}$, A. Athijayamani ${ }^{2, *}$, K. Ramanathan ${ }^{3}$ and S. Sidhardhan ${ }^{4}$ \\ ${ }^{1}$ Dept. of Mechanical Engineering, LMCET, Kanyakumari -629195, Tamilnadu, India \\ E-mail:sujin jose@yahoo.com \\ ${ }^{2, *}$ Dept. of Mechanical Engineering, GCE, Bodinayakkanur-625 513, Tamilnadu, India \\ E-mail: athimania@gmail.com \\ ${ }^{3}$ Dept. of Mechanical Engineering, ACCET, Karaikudi-630 004, Tamilnadu, India \\ E-mail: ramsananthi@gmail.com \\ ${ }^{4}$ Dept. of Civil Engineering, GCE, Tirunelveli-627 007, Tamilnadu, India \\ E-mail: sidhardhant@yahoo.co.in
}

\begin{abstract}
In this study, Wood Dust (WD)/Phenol Formaldehyde (PF) and Coir Pith (CP)/PF composites were hybridized with the Prosopis Juliflora Fiber (PJF) to obtain the hybrid composites. Composites were prepared by hand moulding technique. The weight percentage of particles and fibers are fixed in the ratio of 1:1. Mechanical properties such as tensile, flexural and impact strengths were evaluated as a function of the particle and fiber loadings. The results show that the properties of both the WD and CP composites obviously improved by the addition of the PJF. The improvement in WD/PF composites was obviously higher than the CP/PF composites for all loadings. The WD/PJF/PF hybrid composites exhibited better tensile (strength of $48.9 \mathrm{MPA}$ and modulus of $1262.1 \mathrm{MPa}$, respectively), flexural (strength of $55.4 \mathrm{MPa}$ and modulus of $1344.3 \mathrm{MPa}$, respectively), and impact properties $\left(1.32 \mathrm{KJ} / \mathrm{m}^{2}\right)$.
\end{abstract}

\section{Indexing terms/Keywords}

Prosopis Juliflora fiber, coir pith, wood dust, phenol formaldehyde, physical properties, mechanical properties.

\section{Academic Discipline And Sub-Disciplines}

Polymer Engineering and Technology, Engineering Materials, and Mechanical Engineering;

\section{SUBJECT CLASSIFICATION}

Polymer and material analysis, Mechanical properties, and Effects of hybridization

\section{TYPE (METHOD/APPROACH)}

Experimental analysis

\section{INTRODUCTION}

In recent years, the polymer composite materials reinforced with the fiber and particles are used in many engineering applications (automobiles, structural, sporting goods, marine, electrical, industrial, household and construction) due their high strength and stiffness, light weight and high corrosion resistance ${ }^{1,2}$. Consequently, a continuous improvement in fiber/polymer matrix and particle/polymer matrix, development in manufacturing technology, advanced polymer composite opens a new gateway for the production and development of high performance polymer composites reinforced with the fibers and particulates ${ }^{3}$. Nowadays, natural cellulose fibers and particulates are mostly used as reinforcing agents in thermoset and thermoplastic polymers because they have good constancy between their properties and production costs ${ }^{4}$. However, the properties of fiber or particulate reinforced polymer composites mainly depend on the strength and stability of the interface between the fiber and the matrix. When the interface is weak, fibers or particulates could not act as an effective reinforcing agent in the polymer matrix ${ }^{5}$.

The strength and stiffness of the advanced polymer composites depend on the stress transfer from the reinforcing agents (fibers and particulates) to the polymer matrix and can create by a proper interfacial bonding (some extended chemical compatibility) between the reinforcing agents and the matrix. Therefore, the reinforcing agent-matrix interface plays a major role in the physical and mechanical properties of polymer composite materials. Many efforts have been taken to improve the interfacial bonding between the reinforcing agents and the polymer matrix ${ }^{6}$. The particulates are often added to polymer materials to augment their processability and mechanical properties, as well as to reduce production and material costs. The particulate behavior in the polymer composites was affected by factors like the particle size and shape, distribution, surface area, and surface chemistry. They are acting as a bridge between the fiber and the matrix when they are hybridized with the fiber reinforced polymer composites.

At present, the research of many authors is focused on the enhancement of the mechanical properties of polymer composites by incorporating natural cellulose fibers and bio waste particulates. The evaluation of polymer composites reinforced with the natural cellulose fibers and bio waste particulates has been carried out by the several researchers ${ }^{7}$. Keeping this in view, the Prosopis Juliflora fiber is hybridized with the wood dust and coir pith particulate composites. No 
data are reported on the effects of addition of Prosopis Juliflora fiber in the wood dust and coir pith particulate composites. Hybrid composites are investigated for their mechanical properties and the results are compared with the previous results of wood dust/phenol formaldehyde and coir pith/phenol formaldehyde composites. Efforts are made to investigate the role of the Prosopis Juliflora fiber inclusion on the properties of wood dust/phenol formaldehyde and coir pith/phenol formaldehyde composites.

\section{EXPERIMENTAL}

\subsection{Materials}

A phenol formaldehyde was considered as a matrix material in this study. Wood dust and coir pith particles are used as reinforcing agents for the phenol formaldehyde resin matrix. The Prosopis Juliflora fibers are selected as fibers to hybrid with wood dust and coir pith particulate composites. The average length of the Prosopis Juliflora fibers was approximately $9 \mathrm{~mm}$. The average particle size of wood dust and coir pith was about 800 microns.

\subsection{Specimen preparation}

Before the preparation the particles and fibers are dried in sunlight for 12 hours. Selected compositions of particles, fibers, and resin matrixes are mixed and poured into the hand mould with the size of $150 \times 150 \times 3 \mathrm{~mm}$. Then, the mould is closed and allowed to cure at room temperature.

\subsection{Physical testing}

The density of the hybrid composite is obtained experimentally by a simple water immersion technique using the Archimedes principle. The density of the hybrid composite materials will be obtained from the following equation:

$$
\text { Density }=\frac{w_{d}}{w_{s}-w_{s p}}
$$

where $w_{d}$ is the weight of the sample at completely dried condition, $w_{s}$ is the weight of the sample that soaked in kerosene oil, and $w_{s p}$ is the weight of the sample suspended in the oil through a string. Hardness values of composite specimens are obtained by conducting the tests on a Vickers hardness tester (Leitz, Germany). The Vickers hardness numbers (VHN) of the composite are measured under a load of $L=0.3 \mathrm{Kgf}$ using the following formulas:

$$
\begin{aligned}
& H V=0.1889 \frac{L}{P^{2}} \\
& P=\frac{A+B}{2}
\end{aligned}
$$

where $L$ is the applied load, $P$ is the diagonal of square impression $(\mathrm{mm}), A$ is the horizontal length ( $\mathrm{mm}$ ), and $B$ is the vertical length $(\mathrm{mm})$.

\subsection{Mechanical testing}

The tensile properties are obtained by conducting the tensile tests on the FIE universal testing machine according to ASTM D638 at a crosshead speed of $2 \mathrm{~mm} / \mathrm{min}$ and a gauge length of $50 \mathrm{~mm}$. The flexural properties are observed by conducting the tests on the same machine according to ASTM D790 at a crosshead speed of $2 \mathrm{~mm} / \mathrm{min}$ and a gauge length of $50 \mathrm{~mm}$. Impact property is measured at Izod test machine according to ISO 180.

\section{RESULTS AND DISCUSSION}

\subsection{Physical properties}

Density is one of the most important physical properties in polymer composites and mainly depends on the relative proportion of reinforcing agent and polymer matrix. The densities of the composite specimens measured experimentally are shown in Fig.1. From the Fig.1, It can be seen that the presence of WD particles and PJF fibers as a reinforcing agent in PF matrix reduces the density of the composite up to $20 \mathrm{wt} \%$ and hence makes it lightweight. This may be due to interruption of distribution of reinforcing agents (particles and fibers) and resulting in high void contents. If the void content is high, the density of composites is decreased. The density of composites is increased from 30 wt\% and attains the maximum value at $40 \mathrm{wt} \%$. It may be due to the proper distribution of particles and fibers within the composites. It is also observed that the presence of CP particles and PJF fibers in PF resin matrix reduces the density of the composite up to $50 \mathrm{wt} \%$. It may be due to the high void contents in the CP/PJF/PF hybrid composites. 


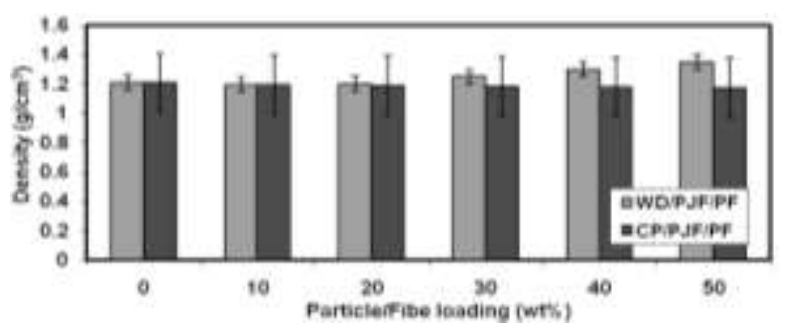

Figure 1. Variations of density of WD/PJF/PF and CP/PJF/PF hybrid composites

Hardness values of WD/PJF/PF hybrid composites are given in Fig.2 . it is observed that the hardness hybrid polymer composites is more than that of the pure PF specimen and also increases with the increase in amount of WD particles and PJF fibers. The increase in hardness of the composite may be due to stronger interface bonding of the WD particle and PJF fibers with PF resin. The hardness values of CP/PJF/PF hybrid composites are shown in Fig.2. It can be understood from the figure that the hardness value increases with an increase in the reinforcing agents (CP and PJF) up to $40 \mathrm{wt} \%$ and then decreases.

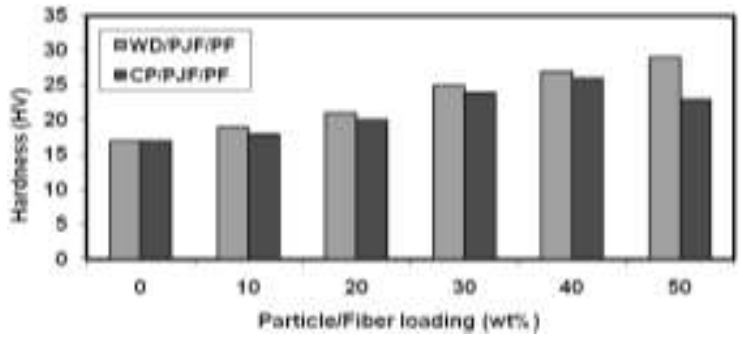

Figure 2. Variations of Hardness of WD/PJF/PF and CP/PJF/PF hybrid composites

\subsection{Mechanical properties}

Mechanical properties provide information about the behavior of the composite material when it is subjected to the external stress before it fails. In this study, the mechanical properties such as tensile, flexure and impact were used to evaluate the strength and toughness of WD/PJF/PF and CP/PJF/PF hybrid composites. By incorporating PJF fibers into the WD/PF and CP/PF composites, the positive effects, as expected were achieved in the mechanical properties. The results revealed that the inclusion of PJF fibers has very strong influence on the mechanical properties of WD/PF composites than that of CP/PF composites.

\section{Tensile properties}

The comparison of tensile properties of hybrid composites is shown in Fig.3. The maximum tensile strength value is obtained at $40 \mathrm{wt} \%$ for both the hybrid composites. The further addition of PJF fibers reduces the tensile strength of hybrid composites. It can be observed that the inclusion of PJF fibers into WD/PF composites at 40 wt $\%$ have increased the tensile strength by $64.1 \%$ and $22.86 \%$, respectively, compared to the neat resin composite and the WD/PF (30 wt\%), but the addition of PJF fibers into CP/PF composites at $40 \mathrm{wt} \%$ has increased the tensile strength by $47.32 \%$ and $22.63 \%$ respectively, compared to the neat resin composite sample and the CP/PF composites. The increase in the tensile properties of WD/PF hybrid composites is associated with the addition of PJF fibers. This may by due to the form of stronger interfacial adhesion between the PF resin matrix, WD particles, and PJF fibers. When compared to CP/PJF/PF hybrid composite, the WD/PJF/PF hybrid composite at $40 \mathrm{wt} \%$ shows $11.39 \%$ of the improvement in tensile strength.

The effect of addition of PJF fibers on the tensile modulus of hybrid composites is also shown in Fig.3. It is evident from the Figure that the tensile modulus increases with the increase in particle and fiber contents at both the hybrid composites. The maximum tensile modulus value is obtained at $50 \mathrm{wt} \%$ for both the hybrid composites. The increase in particle and fiber contents results in the increased brittleness of the both the hybrid composites; thus stress/strain curve of both the hybrid composites becomes steeper. Due to the higher amount of reinforcing agents, the degree of encumbrance increases, which in turn increases the modulus of the composites. When compared to CP/PJF/PF hybrid composite, the WD/PJF/PF hybrid composite at $50 \mathrm{wt} \%$ shows $3.22 \%$ of the improvement in tensile modulus.

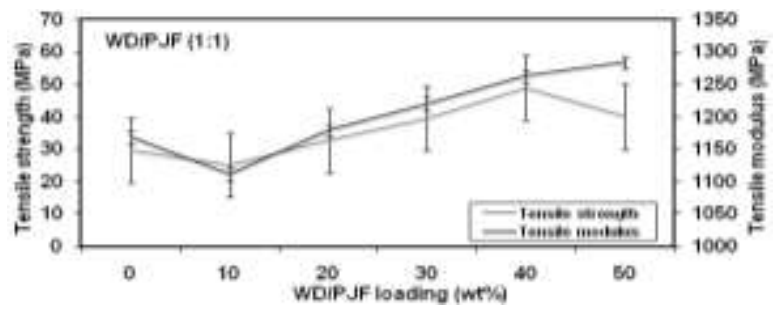

(a)

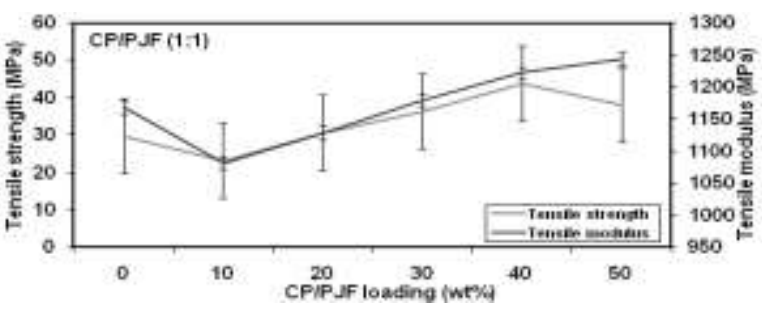

(b)

Figure 3. Variations of tensile properties: (a) WD/PJF/PF hybrid composite and (b) CP/PJF/PF hybrid composite 


\section{Flexural properties}

The effect of addition of PJF fibers on the flexural properties of WD/PF and CP/PF hybrid composites is shown in Fig.4. It is observed from the Fig.4 that the flexural strength of hybrid composites increases with increase in particle and fiber content up to $40 \mathrm{wt} \%$, and then it decreases. It follows the similar trend as tensile strength. The reasons for the lower flexural strength at higher content of the particle and fiber are probably due to the poor dispersion of the particles and fibers in the PF resin matrix, void formation, and weak particle-to-particle, fiber-to-fiber, and also particle-to-fiber interaction. The WD/PJF/PF hybrid composites showed the higher flexural strength values than the CP/PJF/PF, which may be due to the better compatibility between the PJF fibers and WD particles. The WD/PJF/PF hybrid composite shows the higher flexural strength value at $40 \mathrm{wt} \%$, which is $59.37 \%$ and $23.44 \%$ higher than the neat resin sample and WD/PF composites, respectively. In the case of CP/PJF/PF hybrid composites, the maximum flexural strength value is also obtained at $40 \mathrm{wt} \%$. The improvements of $60.47 \%$ and $23.53 \%$ are obtained compared to the neat resin sample and the CP/PF composite. When compared to CP/PJF/PF hybrid composite, the WD/PJF/PF hybrid composite at 40 wt\% shows $14.49 \%$ of the improvement in flexural strength.

The flexural modulus values of WD/PJF/PF and CP/PJF/PF hybrid composites with varying content of WD, CP, and PJF is shown in Fig.4. The flexural modulus values of both the hybrid composites are increased with the increase of the particles and fiber content. The WD/PJF/PF hybrid composites exhibited better flexural modulus than that of CP/PJF/PF hybrid composites. The maximum flexural modulus value is observed at 50 wt $\%$ for both the hybrid composites. When compared to CP/PJF/PF hybrid composite, the WD/PJF/PF hybrid composite at 50 wt $\%$ shows $6.23 \%$ of the improvement in flexural modulus.

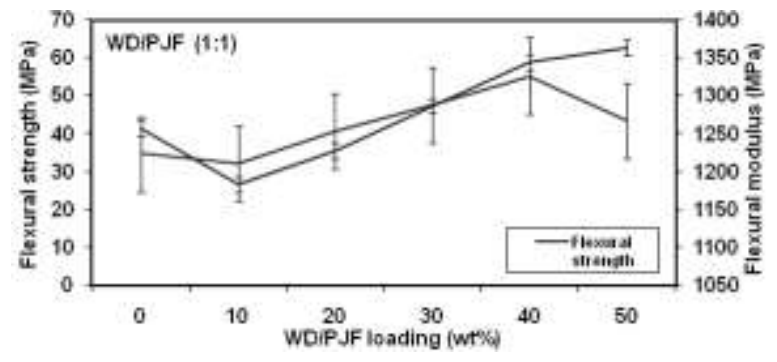

(a)

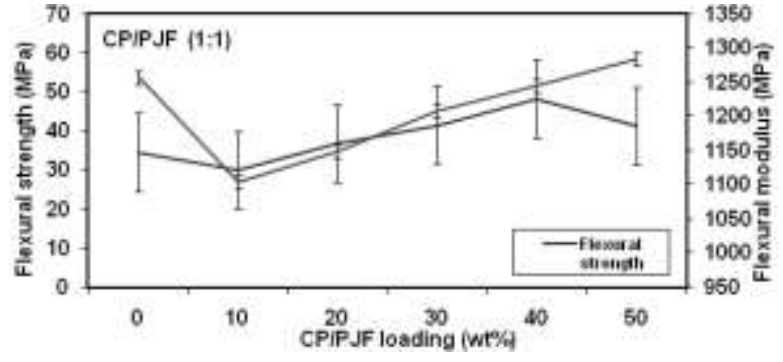

(b)

Figure 4. Variations of flexural properties: (a) WD/PJF/PF hybrid composite and (b) CP/PJF/PF hybrid composite

\section{Impact strength}

Figure 5 shows the effect of addition of PJF fibers on the impact strength of WD/PF and CP/PF hybrid composites. From the Fig. 5, it is observed that the impact strength increases with the increase in PJF fiber content up to $40 \mathrm{wt} \%$ at both the hybrid composites and further it decreases at both the cases. The decrease in impact strength at higher levels of reinforcing content may be due to the probability of PJF fiber agglomeration with the particles. The impact strength of hybrid composites increased with the content up to $40 \mathrm{wt} \%$. The reasons are that the content of fibers and particles is capable of absorbing energy and pressure, which removes the voids contents in the hybrid composites because of the sufficient level of bonding between the particles, fiber, and matrix. The higher level of impact strength of $1.32 \mathrm{~kJ} / \mathrm{m}^{2}$ is obtained for WD/PJF/PF hybrid composites at $40 \mathrm{wt} \%$, which is $3.94 \%$ is higher than that of CP/PJF/PF hybrid composite at same weight percentage.

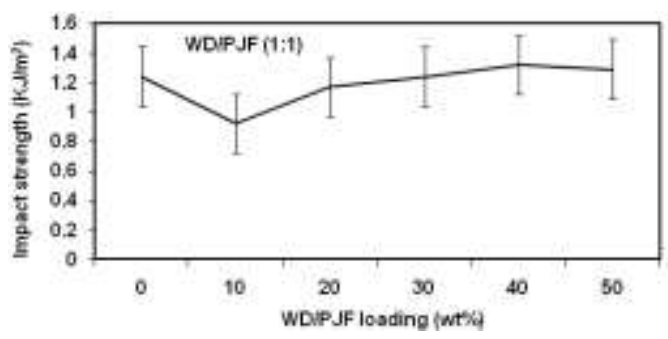

(a)

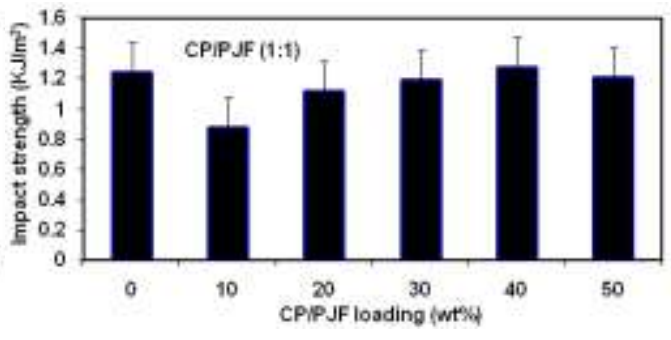

(b)

Figure 5. Variations of impact strength: (a) WD/PJF/PF hybrid composite and (b) CP/PJF/PF hybrid composite

The tensile, flexural and impact strength of hybrid composites increases with an increase in the particles and fiber content up to $40 \mathrm{wt} \%$. However, further increase in the particles and fiber content the strength decreases. This decrease may be due to the improper adhesion inhibit the increase of strength values. As the reinforcing content (particles and fiber) increases, instead of dispersing the gathering of particles and fibers take place and the resin cannot wet the particles and fibers due to non-entrance of resin in-between the two adjacent fibers and particles. The tensile and flexural modulus values increased with increasing the content of reinforcing agents (particles and fiber). The addition of PJF fibers offers a positive improvement in the mechanical properties of WD/PF and CP/PF hybrid composites 


\section{CONCLUSION}

The physical and mechanical properties of the WD/PJF/PF and CP/PJF/PF hybrid composites were evaluated based on the weight percentages of particles and fibers. The properties of the hybrid composites are significantly influenced by the particles and fibers. The density of WD/PJF/PF hybrid composites is increased with the weight percentage of particles and fibers, whereas the density of CP/PJF/PF hybrid composites deceases with the weight percentage of particles and fibers. The hardness of the WD/PJF/PF hybrid composites is increased by increasing the weight percentage of particles and fibers, but the hardness values of the CP/PJF/PF hybrid composites are increased up to $40 \mathrm{wt} \%$ and then deceases. The tensile, flexural, and impact strength values of both the hybrid composites are increased up to $40 \mathrm{wt} \%$ and then dropped. The both the tensile and flexural modulus values increases with the increasing the weight percentage of the particles and fibers. The WD/PJF/PF hybrid composites exhibited better tensile, flexural, and impact properties.

\section{REFERENCES}

1. Dittenber, D.B. and GangaRao, H.V.S. "Critical review of recent publications on use of natural composites in infrastructure", Compos. Part A, Vol.43 (8), pp. 1419-1429, 2011.

2. Zini, E. and Scandola, M. "Green composites: an overview", Polym. Compos., Vol.32 (12), pp. 1905-1915, 2011.

3. Le, M.T. and Pickering, K.L. "The potential of harakeke fibre as reinforcement in polymer matrix composites including modelling of long harakeke fibre composite strength", Compos. Part A, Vol. 76, pp. 44-53, 2015.

4. Efendy, M.G.A. and Pickering, K.L. "Comparison of harakeke with hemp fibre as a potential reinforcement in composites”, Compos. Part A, Vol. 67, pp. 259-267, 2014.

5. Husseinsyah, $S$ and Mostapha, M. "The effect of filler content on properties of coconut shell filled polyster composites", Malaysian polym. J., Vol.6, pp.87-95, 2011.

6. Andrzej, K and Abdullah, A. "Barley Husk and Coconut Shell Reinforced Polypropylene Composites: The Effect of Fiber Physical, Chemical and Surface Properties “, Compos. Sci. Technol., Vol. 70(5), pp. 840-846, 2010.

7. Raju, A G.U., Kumarappa, B S. and Gaitonde, C V.N. "Mechanical and physical characterization of agricultural waste reinforced polymer composites”, J. Mater.Environ.Sci., Vol.3, pp 907-916, 2012.

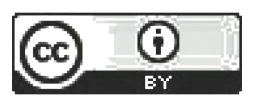

This work is licensed under a Creative Commons Attribution 4.0 International License. 